\title{
Evolution of the Fermi Surface with Carrier Concentration in $\mathrm{Bi}_{2} \mathrm{Sr}_{2} \mathrm{CaCu}_{2} \mathrm{O}_{8+\delta}$
}

\author{
H. Ding, ${ }^{1,2}$ M. R. Norman, ${ }^{2}$ T. Yokoya, ${ }^{3}$ T. Takeuchi, ${ }^{2,4}$ M. Randeria, ${ }^{5}$ J. C. Campuzano, ${ }^{1,2}$ T. Takahashi, ${ }^{3}$ \\ T. Mochiku, ${ }^{6}$ and K. Kadowaki ${ }^{6,7}$ \\ ${ }^{1}$ Department of Physics, University of Illinois at Chicago, Chicago, Illinois 60607 \\ ${ }^{2}$ Materials Sciences Division, Argonne National Laboratory, Argonne, Illinois 60439 \\ ${ }^{3}$ Department of Physics, Tohoku University, 980 Sendai, Japan \\ ${ }^{4}$ Department of Crystalline Materials Science, Nagoya University, Nagoya 464-01, Japan \\ ${ }^{5}$ Tata Institute of Fundamental Research, Bombay 400005, India \\ ${ }^{6}$ National Research Institute for Metals, Sengen, Tsukuba, Ibaraki 305, Japan \\ ${ }^{7}$ Institute of Materials Science, University of Tsukuba, Ibaraki 305, Japan
}

(Received 5 November 1996)

\begin{abstract}
We show, by use of angle-resolved photoemission spectroscopy, that underdoped $\mathrm{Bi}_{2} \mathrm{Sr}_{2} \mathrm{CaCu}_{2} \mathrm{O}_{8+\delta}$ appears to have a large Fermi surface centered at $(\pi, \pi)$, even for samples with a $T_{c}$ as low as $15 \mathrm{~K}$. No clear evidence of a Fermi surface pocket around $(\pi / 2, \pi / 2)$ has been found. These conclusions are based on a determination of the minimum gap locus in the pseudogap regime $T_{c}<T<T^{*}$, which is found to coincide with the locus of gapless excitations in momentum space (Fermi surface) determined above $T^{*}$. These results suggest that the pseudogap is more likely of precursor pairing rather than magnetic origin. [S0031-9007(97)02849-4]
\end{abstract}

PACS numbers: 74.25.Jb, 71.18.+y, 74.72.Hs, 79.60.Bm

One of the first important results obtained from angleresolved photoemission spectroscopy (ARPES) on optimally doped high temperature superconductors was the observation of a large Luttinger Fermi surface containing $1+x$ holes, where $x$ is the hole doping. This has been clearly established now for a number of cuprates including YBCO [1], BSCCO [2,3], and NCCO [4]. This is in contrast to a Fermi surface containing $x$ holes centered about the antiferromagnetic zone boundary which might be anticipated from doping the parent insulator compound. Therefore, an important question is how the optimally doped Fermi surface evolves as the doping is reduced. In the past, only one such study existed [5].

Recently, the Stanford group [6] and our own group [7] have presented data on underdoped BSCCO. These data are quite remarkable in that they show the existence of a highly anisotropic ( $d$-wave-like) pseudogap which persists up to a temperature $T^{*}$ that can be significantly higher than $T_{c}$ [7]. This raises the question whether this pseudogap is a precursor to the insulator gap or a precursor to the superconducting gap.

In this Letter we show that the above two questions are connected. First, for all doping levels studied, we infer a large Luttinger Fermi surface whose volume is consistent with $1+x$ holes. Second, we find that the Fermi surface - the locus of points in momentum space with gapless excitations - above $T^{*}$ coincides with the minimum gap locus - locus of points with minimum excitation gap-in the pseudogap regime $\left(T_{c}<T<\right.$ $\left.T^{*}\right)$. This means that the pseudogap is tied to the Fermi surface rather than to the antiferromagnetic zone boundary, which is in support of a pairing origin for the pseudogap. Third, we find that at low temperatures, there are always two features in the spectra near the $(\pi, 0)$ point of the zone, a low binding energy peak and a higher binding energy hump. It is the leading edge of the former which determines the pseudogap and minimum gap locus, implying that the electronic structure does not change significantly with doping. What does occur is that the intensity of both features is suppressed when reducing the doping, with the low energy peak being suppressed more rapidly than the incoherent hump.

The experiments were carried out at the Synchrotron Radiation Center following procedures described previously $[7,8]$. Underdoping was achieved by adjusting the oxygen partial pressure during annealing of the float-zone grown crystals. These crystals have sharp $\mathrm{x}$-ray diffraction rocking curves with structural coherence lengths similar to the near-optimally doped samples studied earlier. All samples show a very flat surface after cleaving, which is essential for determining the Fermi surface. We have studied several samples ranging from overdoped to underdoped (optimal doping corresponds to a $92 \mathrm{~K} T_{c}$ ); in this Letter we focus on a moderately underdoped sample with a $83 \mathrm{~K} T_{c}$ (transition width $2 \mathrm{~K}$ ), and a heavily underdoped sample with $T_{c}$ of $15 \mathrm{~K}$ (width $>5 \mathrm{~K}$ ), and contrast these results with a slightly overdoped $87 \mathrm{~K}$ sample (width $1 \mathrm{~K}$ ). We will label the samples by their onset $T_{c}$ 's.

Typically in ARPES one determines the Fermi surface by observing the dispersion of a peak through the Fermi energy. Concomitant with this dispersion, the leading edge of the energy distribution curve (EDC) moves to low binding energy, then moves back to higher binding energy, with the moving back due to the drop of spectral intensity as $k$ goes beyond $k_{F}$. Therefore, a simple way to characterize the Fermi surface in ARPES is to track the evolution of the leading edge of the EDC. If all EDCs along a cut in momentum space are plotted on top of one another [see 
(a)

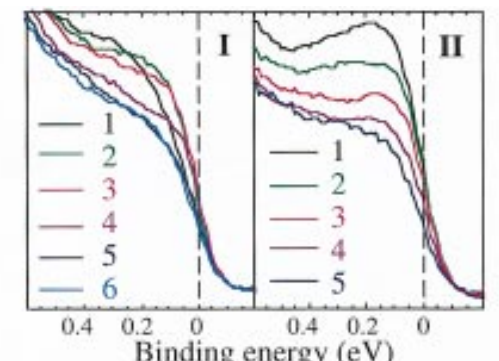

(b)

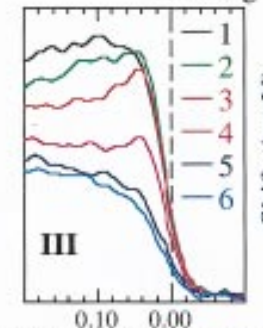

Binding energy $(\mathrm{eV})$

(c)

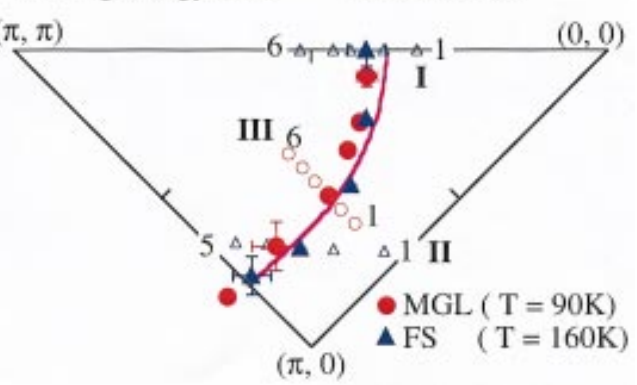

FIG. 1(color). Measurements of the $83 \mathrm{~K}$ sample. (a) EDCs at a photon energy of $19 \mathrm{eV}$ at $160 \mathrm{~K}$ along cuts I and II in (c). (b) EDCs at a photon energy of $22 \mathrm{eV}$ at $90 \mathrm{~K}$ along cut III in (c) (left panel). Midpoint shifts (blue dots) and widths (red diamonds) of this cut (right panel). (c) Fermi surface (FS) at $160 \mathrm{~K}$ (solid blue triangles) and minimum gap locus (MGL) at $90 \mathrm{~K}$ (solid red dots). Cuts I, II (open blue triangles), and III (open red dots) are locations of EDCs in (a) and (b). Notice that the two surfaces coincide within error bars. The error bars represent uncertainties of Fermi crossings as well as possible sample misalignment. The red curve is a rigid band estimate of the Fermi surface.

Fig. 1(a)], then one can clearly observe this effect. This behavior has been verified by simulations which take into account the experimental dispersion and resolution, with the EDC just before the leading edge moves back agreeing with $k_{F}$ to within half the $k$ resolution. The Fermi crossing determined this way typically coincides with the point where the leading edge has the sharpest slope and whose midpoint has moved to the lowest binding energy. This criterion has the advantage of being well defined even when an excitation gap is present, in which case it defines the minimum gap locus. We note that in a superconductor the minimum gap locus coincides with the normal state Fermi surface $[9,10]$. This would not be the case if the gap were due to a charge or spin density wave, since in that case the minimum gap locus is determined by the $q$ vector of the instability, which would only coincide with the Fermi surface if one had perfect nesting.

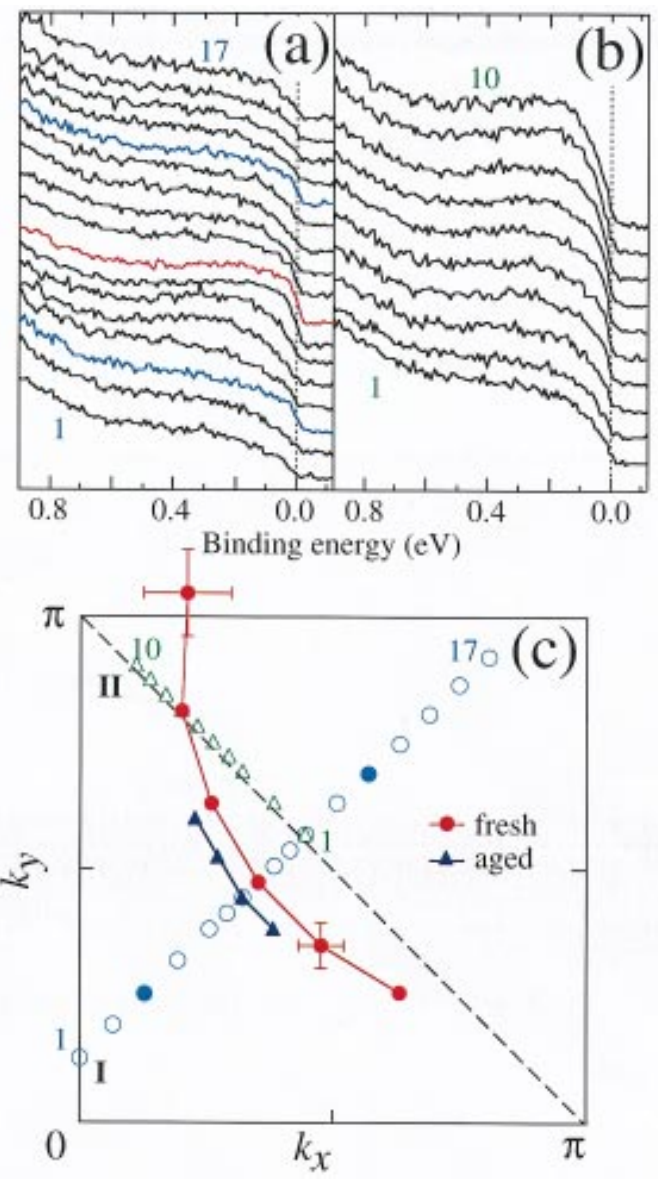

FIG. 2(color). Measurements of the $15 \mathrm{~K}$ sample at a photon energy of $22 \mathrm{eV}$ at $15 \mathrm{~K}$. (a) EDCs along cut I in (c). The red curve is the Fermi crossing, and the blue ones umklapps due to the superlattice [3]. (b) EDCs along cut II in (c). Note that a gap is present for all EDCs from this cut. (c) The minimum gap locus (solid red dots). Solid blue triangles represent the minimum gap locus after the sample has aged, indicating an increased doping [for visual clarity, it is shown only near the $(\pi, \pi)$ direction]. Open dots and triangles represent the cuts from (a) and (b) (with solid blue dots marking the umklapp locations).

In Fig. 1(a) we show EDCs from two representative cuts in the zone for the $83 \mathrm{~K}$ sample at $T=160 \mathrm{~K}$. Since the pseudogap closes at $T^{*} \simeq 170 \mathrm{~K}$ for this sample [7], these data essentially represent the gapless normal state. We note that the spectra are very broad, a consequence of self-energy effects further enhanced by the elevated temperature. In effect, there are no well-defined quasiparticles at this temperature. Despite this, we clearly see dispersion and infer Fermi crossings, where for $k>k_{F}$, the spectral intensity decreases and the leading edge pulls back. The solid triangles plotted in Fig. 1(c) represent our estimate of the Fermi surface which is similar to that for optimally doped samples $[3,8,11]$ and to band theory predictions [12]. Most importantly, we infer a Fermi crossing along the $\bar{M}-Y$ $(\pi, 0-\pi, \pi)$ direction. 
We now turn to the pseudogap state at $90 \mathrm{~K}$, just above $T_{c}$ but well below $T^{*}$. In the left panel of Fig. 1(b) we show that the leading edge evolution is similar to that of Fig. 1(a), the difference being the presence of an excitation gap at $90 \mathrm{~K}$. In the right panel, we plot the midpoint shift of the leading edge of the spectrum and its width along this cut [13]. Both the midpoint shift and the width of the leading edge have a minimum value at the same location along a cut, which defines the minimum gap locus. As shown in Fig. 1(c), this locus coincides with the Fermi surface above $T^{*}$ within experimental error bars.

In Fig. 2(a) we show EDCs from one of several cuts [labeled I in Fig. 2(c)] for the $15 \mathrm{~K}$ sample at $T=15 \mathrm{~K}$, which shows strong dispersive effects even in this highly underdoped sample. The resulting minimum gap locus from these cuts is shown in Fig. 2(c). This locus is similar to that of the $83 \mathrm{~K}$ sample shown in Fig. 1(c), and we find no evidence for a small hole pocket centered about $(\pi / 2, \pi / 2)$. To further test this, we took a cut [labeled II in Fig. 2(c)] along $\bar{M}-Y / 2(\pi, 0-\pi / 2, \pi / 2)$, whose EDCs are shown in Fig. 2(b). If a pocket existed, then one would observe a gapless point along this cut. However a large gap is found at all points, inconsistent with the existence of a Fermi crossing. A plot of the leading edge midpoints along the minimum gap locus is similar to that of a $10 \mathrm{~K}$ sample shown in our earlier work [7], indicating the presence of a highly anisotropic gap. The gap monotonically increases from the $(\pi, \pi)$ direction, but the behavior around the node is flattened relative to a simple $d$-wave gap. This flattening might suggest that the highly underdoped materials actually possess a gapless Fermi arc [6]. However, as explained above, there is no evidence for the closing of this arc, or for a shadow-band feature in the dispersion. A more likely explanation is that the increased linewidth broadening in the underdoped material is responsible for this flattening near the node (e.g., for Lorentzian broadening, the midpoint shift is equal to the actual gap minus $\Gamma$ ), and that this small gap region is just part of a large minimum gap locus which intersects $\bar{M}-Y$. We note that it becomes increasingly difficult to determine the actual minimum gap locus in a region near the $\bar{M}$ point as the sample becomes increasingly underdoped. This is because of the small dispersion near this point, which occurs even for optimal doping, and the strong drop of the signal relative to background emission as the doping decreases. However, our data indicate that the leading edge evolution along cuts near $\bar{M}$ in the underdoped samples is similar to that at optimal doping and consistent with the existence of a large minimum gap locus, which we identify with the Fermi surface underlying the pseudogap state.

In Fig. 3 we plot the Fermi surfaces for the 87, 83, and $15 \mathrm{~K}$ samples. We emphasize that a large surface is found in all three cases, similar to results obtained for underdoped YBCO [5]. Also plotted in Fig. 3 are the predicted surfaces based on a rigid band shift relative to

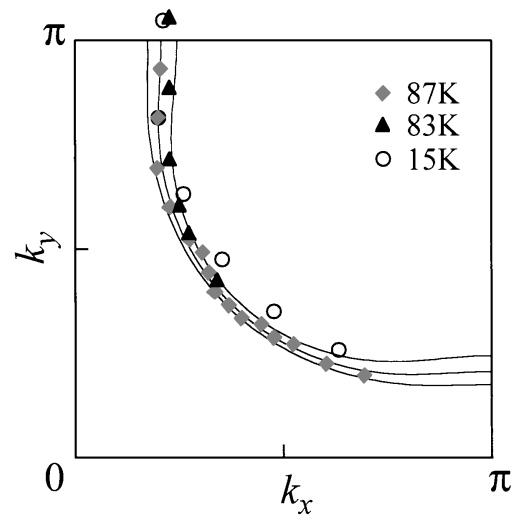

FIG. 3. Fermi surfaces of the 87, 83, and $15 \mathrm{~K}$ samples [error bars are similar to Figs. 1(c) and 2(c). All surfaces have a large volume. The solid lines are tight binding estimates of the Fermi surface at $18 \%, 13 \%$, and $6 \%$ doping assuming rigid band behavior.

the $87 \mathrm{~K}$ sample for doping values of $18 \%(87 \mathrm{~K}), 13 \%$ $(83 \mathrm{~K})$, and $6 \%(15 \mathrm{~K})$. It is apparent from Fig. 3 that the experimental error bars do not permit us to determine the changes in area enclosed by the Fermi surface, and thus to deduce the hole doping $x$. The error bars are due to determining the Fermi crossings as well as the alignment of the samples.

We now turn to the issue of sample aging. BSCCO has a natural cleavage plane due to weak van der Waals coupling of the two $\mathrm{BiO}$ layers. The $\mathrm{BiO}$ surface in optimally doped samples is relatively inert to the residual gases in the vacuum chamber $\left(5 \times 10^{-11}\right.$ Torr $)$, and a clean surface can be regenerated by warming the sample above $50 \mathrm{~K}$. However, we have found this not to be the case in our underdoped samples. In particular, after a day in the chamber, the quasiparticle peak at low temperatures becomes narrower and stronger as illustrated in Fig. 4(a), where we show the spectrum of the $83 \mathrm{~K}$ sample measured at $\bar{M}$ for a newly cleaved surface and after 41 hours, with an $87 \mathrm{~K}$ spectrum plotted for comparison. This is in contrast with expected behavior of surface contamination which would lead to a broader peak due to scattering of the photoelectrons off adsorbed gases. In fact, the spectrum of the aged $83 \mathrm{~K}$ sample becomes similar to that of the $87 \mathrm{~K}$ sample. This leads us to speculate that the underdoped BSCCO surface's doping level has increased. Another important finding from Fig. 4(a) is that the whole spectrum shifts toward the Fermi energy. The shift in the main valence band below $1 \mathrm{eV}$ is about $40 \mathrm{meV}$ which suggests a rigid band shift due to an increase in the doping level. Also, the aging behavior of underdoped BSCCO supports the claim that the broadness of quasiparticle peaks is due to underdoping rather than impurities [7]. Similar behavior is seen in the $15 \mathrm{~K}$ sample, where the aged spectra show a sharper leading edge. In fact, the leading edge behavior of the aged $15 \mathrm{~K}$ sample clearly shows that the Fermi surface 


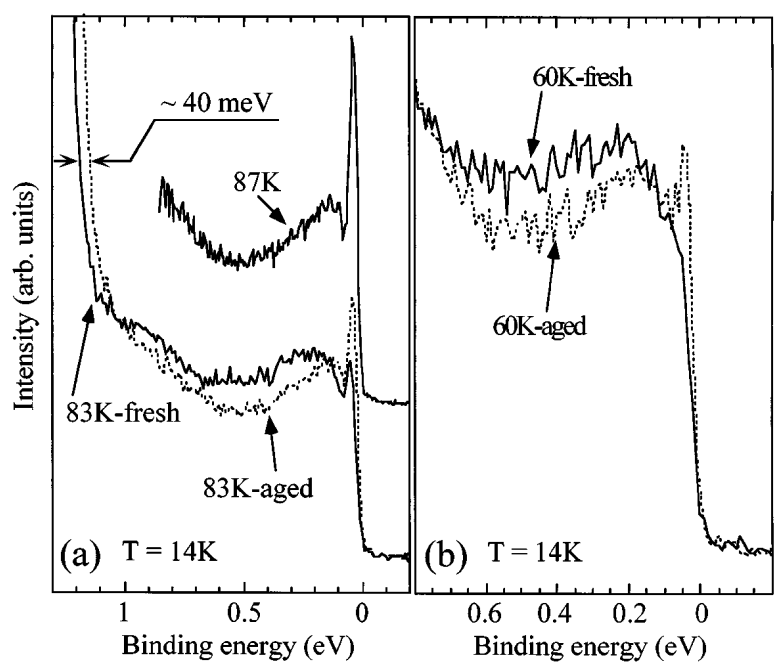

FIG. 4. Aging effects $(T=14 \mathrm{~K})$. (a) EDCs of the $83 \mathrm{~K}$ sample at $\bar{M}$ for a newly cleaved surface and after 41 hours, with the EDC of the $87 \mathrm{~K}$ sample plotted for comparison. The $40 \mathrm{meV}$ shift is between the leading edges of the main valence bands of the two $83 \mathrm{~K}$ spectra. (b) EDC of the $60 \mathrm{~K}$ sample near $\bar{M}$ for an unaged surface and an aged surface. Note the $200 \mathrm{meV}$ hump moves to lower binding energy, and the low binding energy leading edge evolves into a quasiparticle peak.

expands with aging in support of an increase in the doping level, as plotted in Fig. 2. All data presented in Figs. 1-3 are obtained within a short period of time after cleaving, ensuring that intrinsic spectra were measured.

Finally, we consider the theoretical implications of this work. The coincidence of the Fermi surface with the minimum gap locus is in support of a precursor pairing gap interpretation of the pseudogap [14,15], but the present data cannot distinguish between pairing of neutral spinons versus real electrons. We should remark that a simple mean-field simulation of a magnetic gap has a minimum gap location along $\bar{M}-Y$, which does coincide with the paramagnetic Fermi crossing. On the other hand, this gap is around $200 \mathrm{meV}$ if one requires a small hole pocket with the desired size $(x=0.06)$. Now, Marshall et al. [6] do claim the existence of an energy scale at about $200 \mathrm{meV}$ near $\bar{M}$ in underdoped samples. In our opinion, this is associated with the incoherent part of the spectral function. A similar effect is seen in optimally doped samples at low $T$ due to the low frequency suppression of the imaginary part of the self-energy caused by the superconducting gap, which produces a higher binding energy hump in the spectrum separate from the quasiparticle peak [16]. In the normal state, this energy scale is no longer apparent since the gapping effect is removed, and the spectral peak location in the normal state is consistent with a small binding energy (Fig. 2, Ref. [16]). To illustrate this further, we show in Fig. 4(b) data from an underdoped $60 \mathrm{~K}$ sample near $\bar{M}$. The unaged EDC shows a feature near $200 \mathrm{meV}$, but also clearly shows, at a much lower binding energy, a sharp leading edge with a midpoint of $20 \mathrm{meV}$. As the sample ages, the $200 \mathrm{meV}$ feature moves up in binding energy (becoming the hump in optimally doped samples), and the sharp leading edge evolves into a quasiparticle peak [17]. That is, for all doping levels we have studied, there are two features in the spectra near $\bar{M}$, a low binding energy peak and a higher binding energy hump. The former is suppressed rather rapidly with doping, becoming just an edge for sufficiently underdoped samples. Still, it is this feature, rather than the incoherent hump, which determines the pseudogap and minimum gap locus.

In conclusion, we find a large Fermi surface for underdoped BSCCO samples, even with a $T_{c}$ as low as $15 \mathrm{~K}$. No evidence is found for small hole pockets. Moreover, we have shown, at least in an $83 \mathrm{~K}$ sample, that the minimum gap locus in the pseudogap phase coincides with the Fermi surface determined in the normal state above $T^{*}$. This supports the idea that the pseudogap is of precursor pairing rather than magnetic origin.

This work was supported by the U.S. Dept. of Energy, Basic Energy Sciences, under Contract No. W-31-109ENG-38, the National Science Foundation DMR 9624048, and DMR 91-20000 through the Science and Technology Center for Superconductivity, the Japan Society for the promotion of Science, NEDO, and the Ministry of Education, Science and Culture of Japan. The Synchrotron Radiation Center is supported by NSF Grant No. DMR9212658.

[1] J. C. Campuzano et al., Phys. Rev. Lett. 64, 2308 (1990).

[2] C. G. Olson et al., Phys. Rev. B 42, 381 (1990).

[3] H. Ding et al., Phys. Rev. Lett. 76, 1533 (1996).

[4] D. M. King et al., Phys. Rev. Lett. 70, 3159 (1993); R. O. Anderson et al., Phys. Rev. Lett. 70, 3163 (1993).

[5] R. Liu et al., Phys. Rev. B 45, 5614 (1992); 46, 11056 (1992).

[6] D. S. Marshall et al., Phys. Rev. Lett. 76, 4841 (1996); A. G. Loeser et al., Science 273, 325 (1996).

[7] H. Ding et al., Nature (London) 382, 51 (1996).

[8] H. Ding et al., Phys. Rev. Lett. 74, 2784 (1995).

[9] J. R. Schrieffer, Theory of Superconductivity (W. A. Benjamin, New York, 1964).

[10] J. C. Campuzano et al., Phys. Rev. B 53, 14737 (1996).

[11] H. Ding et al., Phys. Rev. B 54, 9678 (1996).

[12] S. Massida, J. Yu, and A. J. Freeman, Physica (Amsterdam) 152C, 251 (1988).

[13] The width of the leading edge is determined by fitting to a Fermi function, the width being the effective temperature of the fit.

[14] M. Randeria et al., Phys. Rev. Lett. 69, 2001 (1992); N. Trivedi and M. Randeria, Phys. Rev. Lett. 75, 312 (1995).

[15] T. Tanamoto, H. Kohno, and H. Fukuyama, J. Phys. Soc. Jpn. 61, 1886 (1992).

[16] M. Randeria et al., Phys. Rev. Lett. 74, 4951 (1995).

[17] We do not see a sharp quasiparticle peak in the unaged spectrum of the $60 \mathrm{~K}$ sample, even at $14 \mathrm{~K}$. This may be due to the very broad transition width of this sample. 